\title{
Enhancements in the structural integrity assessment of plasma facing components
}

\author{
Mike Fursdon ${ }^{a}$, Muyuan Li $i^{b}$, Jeong-Ha You \\ ${ }^{a}$ CCFE, Culham Science Centre, Abingdon, Oxon, OX14 3DB, UK \\ ${ }^{b}$ Max Planck Institute for Plasma Physics, Boltzmann Str. 2, 85748 Garching, Germany
}

This paper provides an overview of analysis methodologies that can be employed to overcome many of the issues associated with the structural integrity assessment of plasma facing components. These issues arise from the multiple materials construction of the plasma facing components (tungsten armour, $\mathrm{CuCrZr}$ heat sink and copper interlayer) making direct application of standard (elastic) structural integrity assessment methods problematic. Example analysis results are used to illustrate these issues, from which it is concluded that PFC structural integrity assessment requires the use of elastoplastic analysis methods, and that this should include: a manufacturing simulation cycle to account for residual stress (and manufacturing strains); pre and post irradiation simulation cycles (with appropriate material models) to correctly assess accumulated ductility usage, and the study of plastic strain in tungsten recrystalised layers to anticipate deep cracking. The issues raised by stress/strain singularities (caused by dissimilar material joints) and multiple ratcheting mechanisms (global, local, material and bi-material ratcheting) are also discussed and proposals made.

Keywords: plasma facing component; structural integrity; monoblock; ratcheting; fatigue;

\section{Introduction}

The typical construction of plasma facing components (PFC) includes tungsten armour, CuCrZr heat sink (e.g. cooling pipe) and copper interlayer. Fig. 1 shows an example ITER-like divertor target "monoblock" PFC design. Improved PFC designs are being developed by EUROfusion to withstand the more demanding environment expected in Demo [1]. Ideally, the merits of a new design are judged initially by structural integrity assessments. This uses finite element (FE) analysis and a structural design code (such as the ITER SDC-IC [2] ) to demonstrate such things as adequate fatigue life and resistance to collapse.
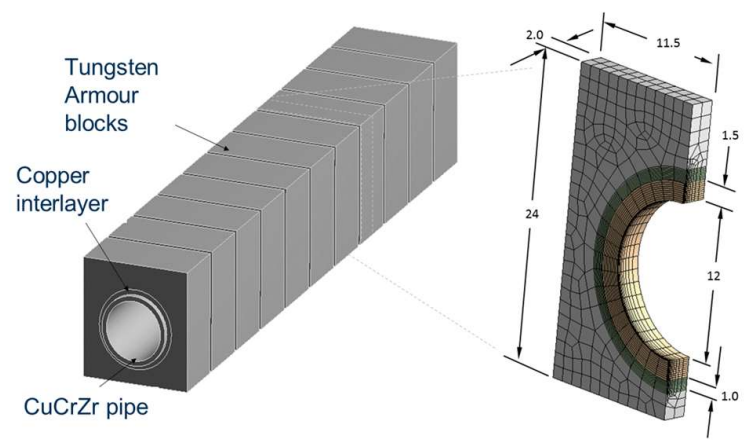

Fig. 1. Monoblock PFC design and typical finite element model used for structural integrity assessment.

Currently most structural assessments are carried out using elastic methods (for example [3]). However, the multiple materials used in PFC construction make these methods problematic because of residual stress, dissimilar material joints and interactions from two plastically deforming materials. This has prompted EUROfusion to conduct a re-appraisal of structural assessment methods with a view to creating a procedure specifically for the structural assessment of PFCs, both in the short term, for current ongoing concept design assessment, and in the long term for final design qualification.

CCFE, as part of EUROfusion's divertor group, are leading the reappraisal of the structural assessment of divertor PFCs. This paper presents an overview of the key issues identified. Example analysis results are presented, and improved assessment methods proposed. The latter include adaptations of existing codes methods, or the creation of new methods from current research. The conclusions are deemed relevant to all PFC designs (and the ongoing Demo Design Criterion development by EUROfusion).

Existing assessments of PFC monoblock designs (for example [3]) tend to study just the $\mathrm{CuCrZr}$ pipe/heat-sink, because only this is perceived as the structural component. However, it is argued that all sub-components must show adequate structural integrity since failure of any one sub-component may easily lead to failure of the whole PFC assembly. The following therefore presents specific methodologies not only for the $\mathrm{CuCrZr}$ pipe but also for the copper and tungsten armour.

\section{Proposed methodologies}

\subsection{Residual stress estimation}

In SDC-IC it is necessary to show that the strain created during all operating conditions does not exceed material rupture-strain limits. However, previous work[4] has shown that thermal cycles in the manufacturing of 
PFCs can create significant residual stress, and since residual stress is created by plastic strain, these strains may also be significant. Therefore, it is proposed that for PFC assessment, manufacturing strains should be included in the structural assessment calculation. (similar to the inclusion of "forming strain" in ASME code [5])

The simplest (and perhaps only) realistic FE method of calculating both manufacturing and operational strains in a single calculation is by elastoplastic analysis, where the required operational load step calculation is preceded by a manufacturing cycle simulation (as shown by [6]).

The manufacturing cycle to be simulated typically includes both a joining process (e.g. by HIPing or braising) and a subsequent heat treatment cycle (soak at $\sim 470^{\circ} \mathrm{C}$ to harden $\mathrm{CuCrZr}$ ). However, it is argued that for assessment, stresses generated during the joining process (which can be difficult to calculate) are limited because the $\mathrm{CuCrZr}$ is in an annealed (soft) state. This means that the final residual stress can be estimated by simulating just the heat treatment cycle (when the $\mathrm{CuCrZr}$ achieves its full strength), and more specifically: the cooling from the soak temperature. The validation of this assumption is ongoing.

Fig. 2 shows an example application of the proposed method on a monoblock with calculated strain evolution for the manufacturing cycle followed by an operations "slow transient" $20 \mathrm{MW} / \mathrm{m}^{2}$ heat load step. The model and materials data for this (and all) simulations in this paper is detailed in reference [4]. The results illustrate that manufacturing strains are significant, and that in this case, the allowable strain (here corrected for triaxial stress effects) is exceeded during the slow transient event. It follows that the proposed assessment methodology exposes a structural integrity fault in this design indicating that modifications are required. The contour plots show that considerable strain concentration occurs in the gap between tungsten blocks. It is suggested that modification of PFC geometry in this area could reduce this concentration so that accumulated manufacturing and operations strains fall within the allowable levels indicated.

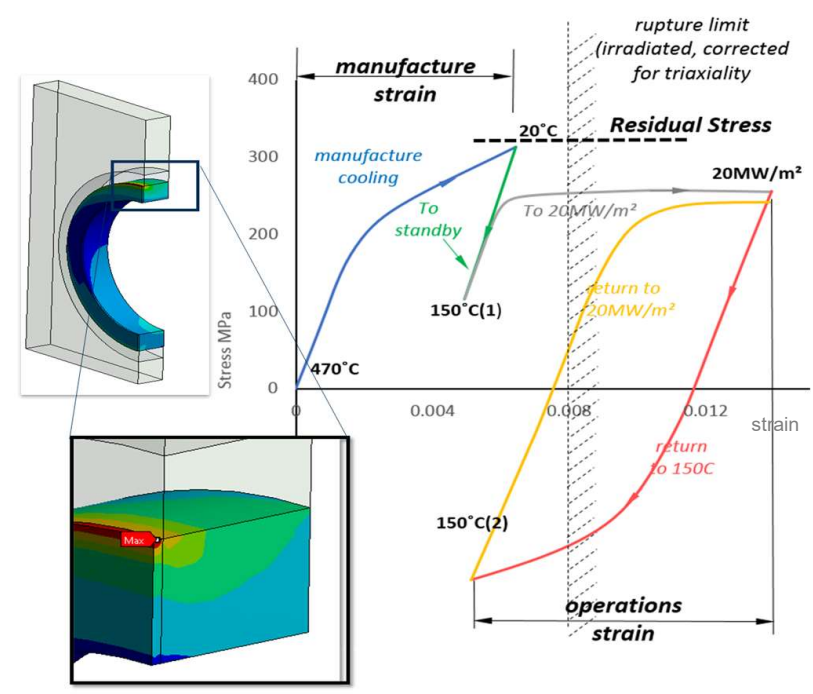

Author's email: mike.fursdon@ukaea.uk
Fig. 2. Analysis of strain evolution at a strain concentration during manufacturing and operational cycles relative to the expected (triaxial stress corrected) rupture limit.

\subsection{Strain singularities}

Typically, in the current design of PFCs, the surface at the copper tungsten joint is flat/flush. Such dissimilar material joints are known for creating singularities in stress/strain analysis [7], preventing valid assessment. To overcome this issue two solutions are proposed:

1) Adopt the existing "hot spot" methods used for the fatigue life assessment of welded joints (e.g. as used in EN13445 [8]). Here a surface stress value is determined by quadratic extrapolation from subsurface stress at three sampling points. The value is used to estimate the fatigue life by comparison with reference test samples with the same "hot spot" stresses.

2) Alternatively, it is suggested that a singularity indicates potential for very high "real" stress of unknown magnitude, and as such, should be avoided by design. Previous studies [7] have shown geometric conditions which prevent dissimilar material joint singularities.

Fig. 3 illustrates a comparison of the calculated hotspot stress for the copper-tungsten joint in a conventional monoblock design against a modified design without singularity. The singularity is removed by changing the angle of intersection of the copper and tungsten surface. Unlike the hot-spot stress, the "real" stress of the modified design can be assessed against existing strain life data, and so enables immediate fatigue life assessment.

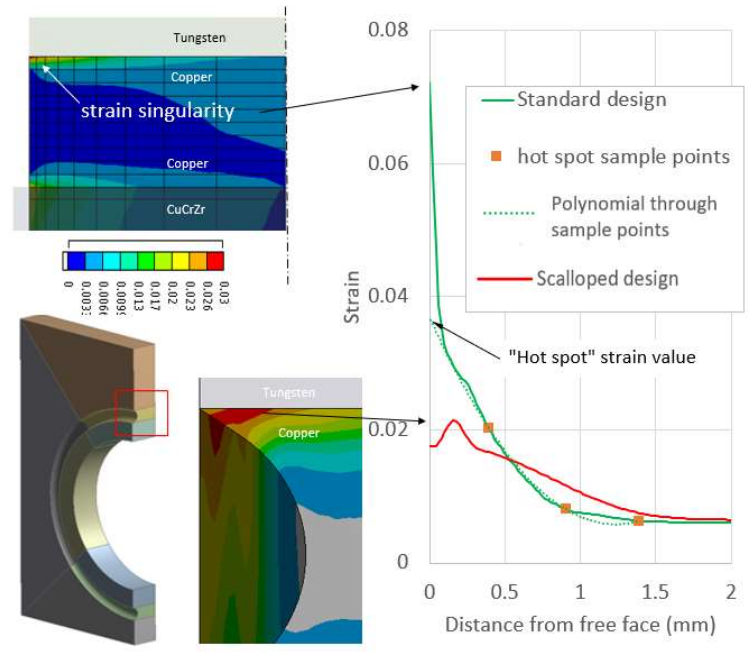

Fig. 3. Strain at the copper tungsten interface showing comparison of example FE calculated strain with standard geometry (with singularity), the associated hot spot strain values and the strain value with an alternative "scalloped" design without singularity.

\subsection{Irradiation hardening}

The most taxing test of a PFC design is its ability to operate under irradiated conditions. In the structural assessment of irradiated PFCs two factors have to be considered:

1. Allowable stress and strains are generally reduced [9] (i.e. are more restrictive) 
2. Stresses are generally increased due to irradiation hardening (as described below).

This simultaneous change in both strain level and allowable strain at the various stages of component life (from unirradiated to irradiated) makes assessment of accumulated ductility usage potentially complex. For this reason, it is proposed that assessment calculations should include time dependent material models (or at least a twostate models) to match the changing stress strain characteristics.

Stress strain data to create these material models (for irradiated condition) is scarce. Most is for monotonic loading (for example [10][11]), whereas many of the structural integrity assessments study cyclic loading conditions. Nonetheless existing data indicates significant hardening occurs (Fig. 4) and so must be included for a valid structural assessment to be made.

To facilitate the immediate application of proposed assessment method, (so that current EUROfusion concepts can be assessed immediately) extrapolations from existing data have been generated. Fig. 4 shows example test data used for this extrapolation, in this case on the significant hardening effects of irradiation on copper. Fig. 4. also shows the results of an example application of the proposed analysis method demonstrating the expected change in interlayer stress, pre and post irradiation. In ANSYS this methodology is achieved by issuing the "MPCHG" command (in this case at step 6 of the analysis) to switch abruptly from unirradiated to irradiated materials properties data predefined.

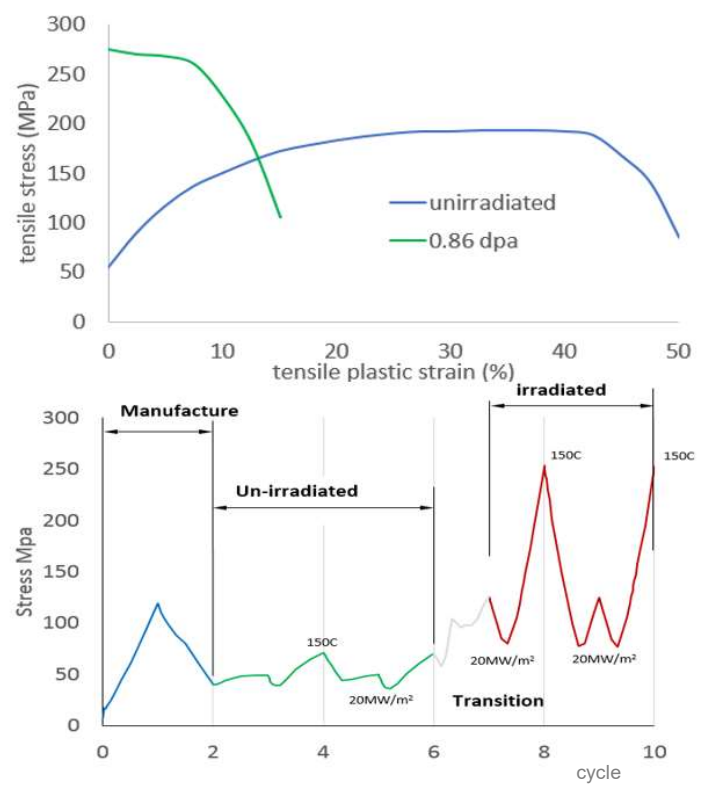

Fig. 4. Stress strain data for irradiated and unirradiated copper [11], and the calculated evolution of monoblock interlayer stress before and after irradiation.

\subsection{Multi-material plasticity interaction and ratcheting}

Ratcheting is the progressive growth of plastic strain zones due to cyclic loading. Two independent failure mechanisms from ratcheting are possible:

1. Plastic collapse due to "global" ratcheting (moderate/high plastic strains over large volume).

2. Exhaustion of ductility due to local ratcheting (localised high plastic strain) by:

a. Material ratcheting (some aspects of which are covered by the Chaboche material model [12]).

b. Multi-material interaction (e.g. by temperature dependent yield stress as described by [13])

In PFCs both the structural material (e.g. CuCrZr) and the interlayer may experience plastic strains (Fig. 5.) leading to interactions that can affect any ratcheting response. This is further reason for proposing the use of elastoplastic analysis in PFC structural assessment. Fig. 6 for example shows the calculated ratcheting effects when an assembly of two different plastically deformable materials undergoes cyclic heating (calculated by a two-bar system model described in the Appendix).

Current elastic code assessment requires only the study of global collapse. Pending further development of material models, this is also seen as the prime objective of elastoplastic assessments. In elasto-plastic analysis, ratcheting is assessed by studying the cycle by cycle evolution of strains. However, the effects of local ratcheting can make the identification of convergent conditions in a global ratcheting assessment problematic. To overcome this issue a method that studies the global ratcheting and local ratcheting process independently is proposed:

1. Global ratcheting is made independent of local ratcheting effects by using elastic perfectly plastic material with single yield value for all temperatures.

2. Local ratcheting is studied with full temperaturedependent Chaboche model (Although failure by this mechanism is yet to be validated).

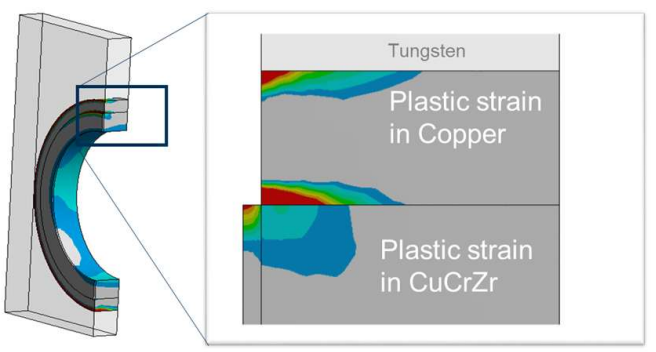

Fig. 5. calculated plastic strain regions in copper and $\mathrm{CuCrZr}$ (at $20 \mathrm{MW} / \mathrm{m}^{2}$ heat load) 


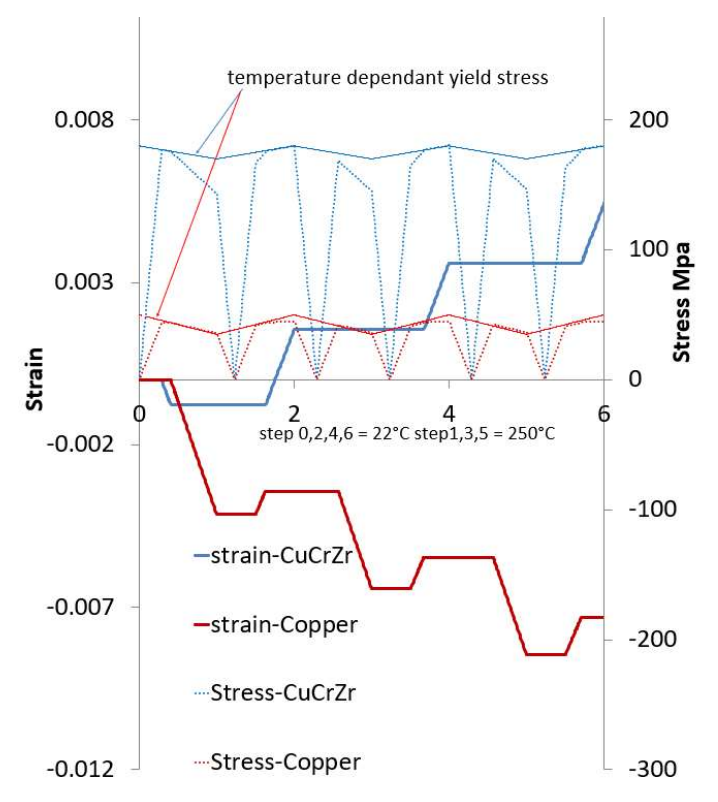

Fig. 6. Two bar study of Copper/CuCrZr plastic strain interaction under temperature cycling alone showing the calculated stress vs the temperature dependent yield stress and the resulting strain ratcheting response.

\subsection{Tungsten assessment}

Ideally the use of brittle materials in load bearing structures should be avoided, and to the authors knowledge, such applications are not covered by existing design codes. For this reason, to make the deemed necessary structural assessment of tungsten in PFCs, new rules are proposed.

Although, it is accepted that probabilistic methods may be the most valid method of brittle material assessment in the long term (as discussed in [14]), these methods are not yet fully validated. Hence, initially, two relatively more conventional design criteria are proposed:

1. The Rankine criterion (maximum principal stress $<$ ultimate tensile strength) with 0.5 safety factor).

2. Fatigue crack initiation (based on the failure modelling of deep cracking by Li et al [15] ).

In the latter, the methodology requires potential recrystalised material to be identified by prior thermal analysis (shown by any material that experiences $>1300^{\circ} \mathrm{C}$ ). This material is assigned elastoplastic properties and the plastic strain during an operating plasma heating cycle is studied (Fig. 7). Strain increments occurring above and below the ductile to brittle transition temperature (DBTT) are assessed independently, and existing strain life curves are used to calculate a crack initiation life. (This is also deemed the effective total life since $\mathrm{Li}$ [15] showed that immediate deep cracking by fast fracture would follow).
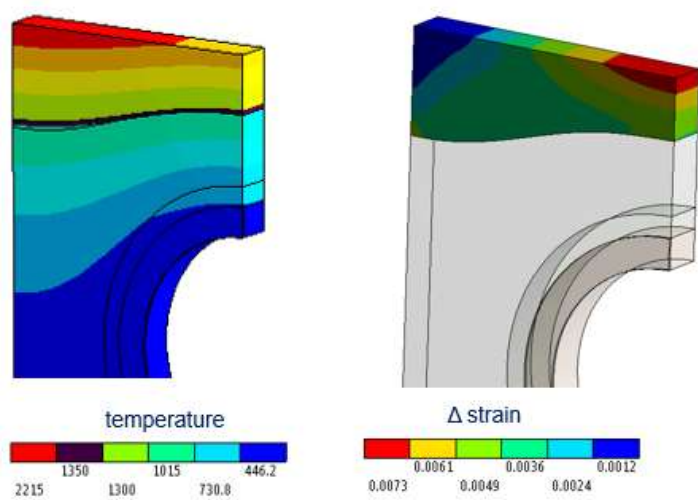

Fig. 7. Thermal analysis to identify potentially recrystallized material (material $>1300^{\circ} \mathrm{C}$ ) and subsequent plastic strain increment assessment below the DBTT.

\section{Summary and Conclusions}

The EUROfusion consortium has identified the need for improved methods to assess the structural integrity of plasma facing components. Following a range of ongoing analysis studies in the consortium's divertor group, it is initially concluded that PFC assessments require the following:

1. Use of elastoplastic methods.

2. Assessment of all subcomponents, i.e of pipe, armour and interlayer.

3. The inclusion of a manufacturing simulation cycle to account for residual stress and manufacturing strains.

4. Analysis of the evolution of strains from the unirradiated to irradiated state for example by using bi-state material models.

5. The separation of global ratcheting from local ratcheting by appropriate material models.

The following is also recommended:

6. Hot-spot methods or joint redesign to address singularities at dissimilar material joints.

7. Assessment of tungsten deep cracking using the methods developed by Li [15] .

\section{Acknowledgments}

This work has been carried out within the framework of the EUROfusion Consortium and has received funding from the Euratom research and training programme 20142018 under grant agreement No 633053 and from the RCUK Energy Programme [grant number EP/I501045] The views and opinions expressed herein do not necessarily reflect those of the European Commission.

Acknowledgment is also given for the help provided by $\mathrm{V}$ $\mathrm{K}$ Thompson (CCFE) in demonstrating multi-material ratchetting effects. 


\section{References}

[1] J.-H. You, et al., European DEMO divertor target: Operational requirements and material-design interface, Nucl. Mater. Energy 9 (2016) 171-176.

[2] ITER design code, In-vessel Components, _SDCIC_222RHC_v3_0.

[3] A Li-Puma et al., Potential and limits of water cooled divertor concepts based on monoblock design as possible candidates for a DEMO reactor. Fusion Engineering and Design Volume 88, Issues 9-10, October 2013, Pages 1836-1843.

[4] Fursdon et al., A hybrid analysis procedure enabling elastic design rule assessment of monoblock-type divertor components. Fusion Engineering and Design 135 (2018) 154-164.

[5] ASME SectionVII Div. 2 Part 5 (5.3.3).

[6] M. Miskiewicz, J.-H. You. Impact of plastic softening of over-aged $\mathrm{CuCrZr}$ alloy heat sink tube on the structural reliability of a plasma-facing component, Fusion Eng.Des. 83 (2008) 66-71.

[7] PA Kelly. The design of joints between elastically dissimilar joints I Mech E Journal of strain 1992.

[8] BS EN13445 British standards institute.

[9] SDC-IC Appendix_A, Materials Design Limit Data (G 74 MA 8 01-05-28 W 0.2) 2012.

[10] P. Fenici, et. al., 'Effect of fast-neutron irradiation on tensile properties of precipitation-hardened $\mathrm{Cu}-\mathrm{Cr}-\mathrm{Zr}$ alloy" Journal of Nuclear Materials (1994).

[11] S.A. Fabritsiev et al., 'Effect of the irradiation-annealingirradiation cycle on the mechanical properties of pure copper and copper alloy" Journal of Nuclear Materials 324 (2004) 23-32.

[12] R Halama et al., Phenomenological Modelling of Cyclic Plasticity. www.intechopen.com

[13] V. V. Silberschmidt, et al., On material immanent ratcheting of two-phase materials under cyclic purely thermal loading. Archive of Applied Mechanics (1999).

[14] D Rubesa et al., Main features of designing with brittle materials Journal of Materials Engineering and Performance, April 2003, Volume 12, Issue 2, 220-228.

[15] Li et al., Interpretation of the deep cracking phenomenon of tungsten monoblock targets observed in high-heat-flux fatigue tests at $20 \mathrm{MW} / \mathrm{m} 2$. Fusion Engineering and Design 101 (2015) 1-8.

\section{Appendix: 2-bar 2-material model}

The material ratcheting effect shown in fig 6 was determined by the 2-bar system shown in fig A1 with elastic perfectly plastic Copper and $\mathrm{CuCrZr}$ bars with 5:1 area ratio set between fixed ends. The bars have dissimilar temperature dependent yield strengths as detailed in table A1. And the system is subjected to repeated isothermal temperatures steps of 20 to 250.The results indicates the potential interaction between the plastic strains in the monoblock $\mathrm{CuCrZr}$ pipe stress concentration and plastic strains in the copper interlayer.

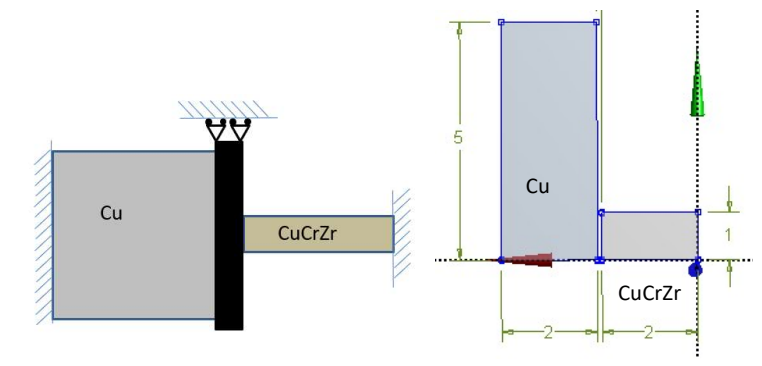

Fig A1 showing schematic of 2 bar system and ANSYS 2 element plane stress model implementation.

\begin{tabular}{ccccc} 
& $\mathrm{T}\left({ }^{\circ} \mathrm{C}\right)$ & $\begin{array}{c}\mathrm{CTE}(\mu- \\
\text { strain })\end{array}$ & $\mathrm{E}(\mathrm{Gpa})$ & $\begin{array}{c}\sigma_{\mathrm{y}} \\
(\mathrm{Mpa})\end{array}$ \\
\hline \multirow{2}{*}{ CuCrZr } & 0 & 16 & 120 & 200 \\
& 400 & 16 & 120 & 160 \\
Copper & 0 & 16 & 120 & 50 \\
& 400 & 16 & 120 & 20
\end{tabular}

